\title{
The RHEA-project robot for tree crops pesticide application
}

\author{
Marco Vieri, Riccardo Lisci, Marco Rimediotti, Daniele Sarri \\ Department of Agricultural, Food Production and Forest Management University of Florence \\ Firenze, Italy
}

\begin{abstract}
The sustainable use of pesticide and the need of a renewed integrated system of agricultural knowledge and management, focus the designing of the EU FP7 RHEA Project. The objectives are the design, development, and testing of a new automatic generation of robotic systems to perform field operations for the sustainable crop management. The project affects three case study: chemical, physical, mechanical and thermal effective weed management in maize and wheat cultivations and chemical pesticide management in woody crops. To achieve the goals, a fleet of small and heterogeneous robots, ground and aerial, equipped with advanced sensors, innovative end actuators and decision control algorithms were realized. Present work is related to the third case study considered i.e. the spraying in woody crops specifically in olive trees. The final decision on woody perennial crops treatment device system, was oriented toward a complete double side air blast sprayer with eight separate spraying modules on four vertical bands of the canopy. Rhea air blast sprayer introduces some important innovations in the studies concerning the pesticide variable rate treatment, i.e. the management possibility of air flow in site specific way and in real time in function of the target.
\end{abstract}

\footnotetext{
Correspondence: Marco Vieri, GESAAF Department of Agricultural, Food Production and Forest Management, Scuola di Agraria, University of Florence, Piazzale delle Cascine 15, 50144 Firenze, Italy.

Tel. +39.055.3288320 - Fax +39.055.331794.

E-mail:marco.vieri@unifi.it

Acknowledgments: authors wish to acknowledge the Nobili.
}

Key words: precision farming, sustainability, variable rate treatment, airblast sprayer, robot.

Contributions: the authors contributed equally.

Conflict of interests: the authors declare no potential conflict of interests. Funding:

Dedication: none

(C) Copyright M. Vieri et al., 2013

Licensee PAGEPress, Italy

Journal of Agricultural Engineering 2013; XLIV(s2):e71

doi:10.4081/jae.2013.s2.e71

This article is distributed under the terms of the Creative Commons Attribution Noncommercial License (by-nc 3.0) which permits any noncommercial use, distribution, and reproduction in any medium, provided the original author(s) and source are credited.

\section{Introduction}

Our society is evermore demanding lower impacts on environment and higher standards in food safety. The new Community rules on the risks reduction to the environment and food safety require a breakthrough innovation in all sectors especially in agriculture at which there is a strong pressure due to the remarkable use of chemicals. The sustainable use of PPP (Plant Protection Products) (Vieri \& Spugnoli, 1996), the need of a renewed integrated system of agricultural knowledge and management, focus the designing of the EU FP7 RHEA Project (Robot fleets Highly for Effective Agriculture and forestry management).

The objectives of the RHEA project are the design, development, and testing of a new automatic generation of robotic systems to perform field operations for the sustainable crop management. To achieve this goal, a fleet of small and heterogeneous robots - ground and aerial equipped with advanced sensors, innovative end actuators and decision control algorithms was realized. RHEA can be considered a cooperative robotic system, falling within an emerging area of research and technology.

Six integrated modules make up the RHEA system: Mission Manager (MM), Perception System (PS), Communication and location System (CS), Actuation System (AC) divided into High Level and Low Level Decision, Mobile Units (MUs) and the Base Station and Graphic User Interfaces (GUI) (Gonzales et al., 2011). The project affects three case study: chemical, physical, mechanical and thermal effective weed management in maize and wheat cultivations and chemical pesticide management in woody crops. Present work is related to the third case study considered i.e. the mobile unit development for spraying in woody crops specifically in olive trees. The design involved the analysis of the following variables:

- Equipment configuration; single side or double side and the number of modules;

- Device system (DS) and the control system or Low Level Actuation System (LLAS);

- Main parameters controlled: spray cloud features, liquid flow rate, air flow rate and the air jet variable direction.

\section{Materials and methods}

The initial project proposal about the RHEA ground mobile units (GMUs) configuration, took into account very small vehicles with mass of 200-400 kg and less than $15 \mathrm{~kW}$ power, operating at a forward speed of $1.5 \mathrm{~m} \mathrm{~s}^{-1}$ with only one operating arm. This setting, is really feasible only for spot spraying technique e.g. in the insects control, but it is not appropriate for other diseases like fungi etc. .In these cases, at least, two problems arise: the first one is the dosage that, even in a modern intensive tree plant with an average of $5000 \mathrm{~m}^{2}$ of canopy surface per hectare, requires not less than 100-200 $\mathrm{l} \mathrm{ha}^{-1}$. The second is the necessity of an air assisted device to optimize the distribution inside the 
canopy. Furthermore, the use of one single spray diffuser, at the prescribed forward speed, produces an unacceptable unequal sinusoidal application. Because of these issues, the RHEA Consortium approved a more suitable ground mobile unit with other new specifications: $4 \mathrm{x} 4$ wheel drive, CVT transmission, $37.3 \mathrm{~kW}$ gross power, with a 10\% of which available as electric power, maximum mass of $1600 \mathrm{~kg}$, three hitch points lift and standard $52 \mathrm{rad} \mathrm{s}^{-1}$ p.t.o. . These new features, make it possible to adopt a common ready to use and innovative air assisted sprayer.

Another important choice, was the scenario for the final demonstration trials: the RHEA Consortium approved an intensive olive plantation. This decision was taken because the olive growing is, at the same time, quite similar to modern orchard crops and woody tree crops. The plantation pattern (Figure 1) was $4.0 \mathrm{~m}$ inter-row and $1.5 \mathrm{~m}$ distance on the row to reach a foliar wall as flat and regular as possible.

With these variables, different solutions about pesticide spraying and air vector devices management were investigated (Figure 2). The final judgment was oriented toward a complete double side air blast sprayer (based on Nobili Oktopus air blast sprayer) with eight separate spraying modules on four vertical bands of the canopy. For the machine automation, three types of kinematic coupling driven by stepper motors were built. These are managed by the motion controller, which allows an accurate motor control both in speed and position. Moreover, they are fully programmable and they can be interfaced with a standard P.L.C. (Programmable Logic Controller). A detection system consisting of eight ultrasonic sensors permits to reach data about the canopy width of each vertical band. Finally, actuation rules (Figure 3) for every one device to better fit optimum spray features (air and liquid flow rate) on each vertical bands of the canopy were defined.

\section{Results}

The design has led to the development of an air blast sprayer (Figure 5); with the following features:

maximum height of the vertical boom $2.5 \mathrm{~m}$;

canopy band to be treated $2.7 \mathrm{~m}$ (3.0 - $3.5 \mathrm{~m}$ maximum crop height);

total equipment mass (empty tank) $400 \mathrm{~kg}$;

tank $300 \mathrm{~L}$;

hydraulic pump with maximum flow rate of $100 \mathrm{~L} \mathrm{~min}^{-1}$ at $25 \mathrm{bar}$ and maximum $5 \mathrm{~kW}$ of power requirement to the p.t.o. ;

fan maximum $15 \mathrm{~kW}$ to the p.t.o.;

- the equipment is semi-loaded and coupled at the three hitch point lift but when it is working, floating and resting on its wheels.
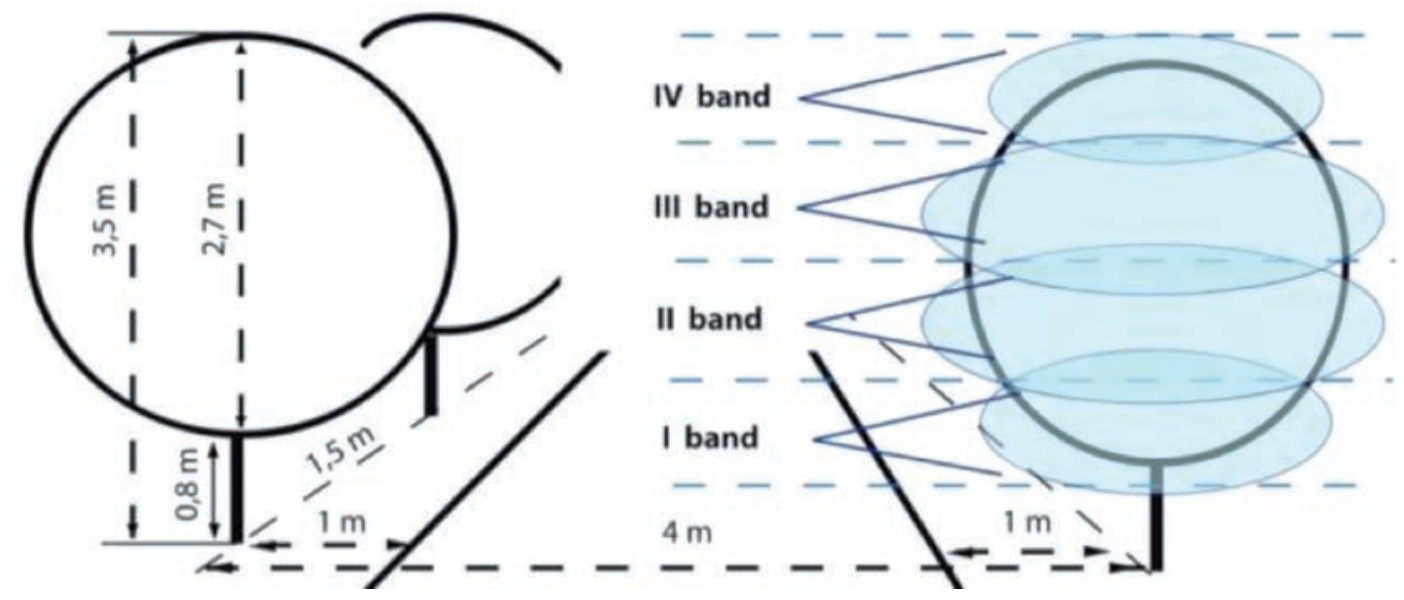

Figure 1. Demonstration woody perennial plant and definition of the four horizontal canopy bands.
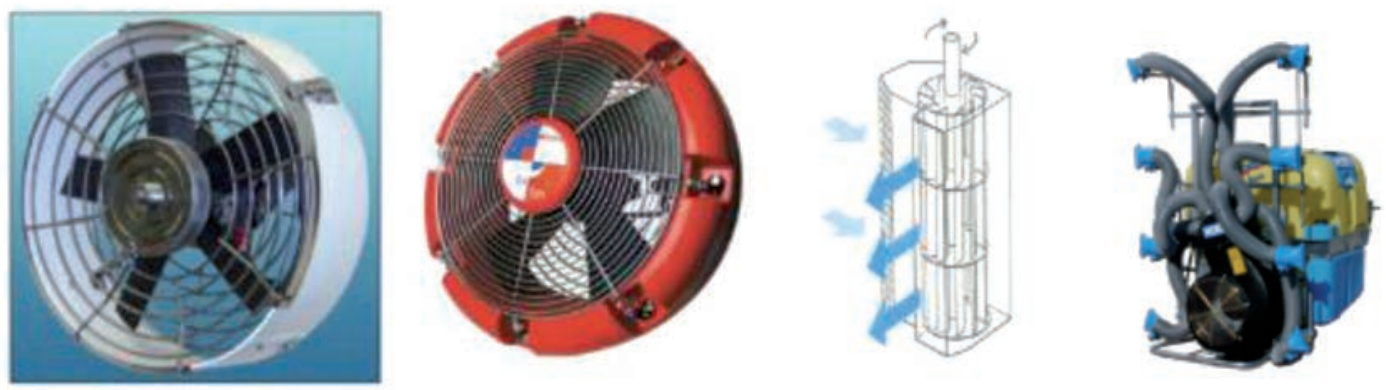

Figure 2. Spraying and air vector devices solutions evaluated for the air blast sprayer configuration: left to right; the Proptec rotary atomizers which use mechanical centrifugal force to atomize fluids; The Sardi fan Multiple air-assisted sprayers, with a series of spray heads with a direct blast axial flow fan and a series of hollow cone nozzles; The tangential cross-flow fan; The Nobili Oktopus sprayer with eight spraying modules manually adjustable. 
In view of the objectives, and starting from the basic configuration of the Nobili Oktopus air blast sprayer, the following changes were designed: detection system composed of eight ultrasonic sensors to reach data on canopy width of each vertical band;

variable control of the liquid flow rate in each module to adapt dosage at the canopy thickness on each band with the following rules: $100 \%$ canopy thickness $100 \%$ dose; $50 \%$ canopy thickness $70 \%$ dose; < $50 \%$ canopy thickness $30 \%$ dose, absence of canopy 0 dose;

- to control flow rate two solutions were developed and tested: an intermittent spray nozzle driven by frequency and duty cycle electronically managed and double nozzles on each module with $70 \%$ and $30 \%$ of needed flow rate on each band simultaneously open with full canopy (Figure. 5);

for the air flow adjustment in the main entrance a fan inlet manifold throttle valve controlled by a stepper motor was built (Figure 4);

the variable inclination (step motor controlled) of the four terminal modules (top and bottom) to improve the deposition in these sensitive areas of the canopy (Figure 4);

to control the airblast flow rate on each module, eights butterfly valves (step motor controlled) located on each pipe of the eight fan calotte collector were designed (Figure 4);

The entire equipment, with the whole system of devices (DS), was monitored by the LLAS that consist in the PLC (Figure 5) and related algorithms. All this is controlled, in its turn, by the HLDMS and upstream from the MM of the RHEA system.

\section{Conclusions}

The Rhea air blast sprayer introduces an important innovation in the studies concerning the pesticide variable rate treatment, i.e. the management possibility of air flow in site specific way and in real time in function of the target. The innovative devices developed and the wide versatility of the actuators designed, makes it possible to adapt the air flow in function of the canopy thickness. The spraying configuration designed provides eight different vertical bands of independent treatment with the possibility to manage, in a site specific way, the pesticide dose applied and the air flow direction and its rate. The spray robot module has both remote and proximal controls; remote for tractor control and proximal for spraying. This choice has a double aim i.e. to have an innovative sprayer usable and testable as independent autonomous equipment or else, coupleable with standard tractors. The expected pesticide dosage saving is about $50 \%$ of the conventional application rate maintaining, at the same time, the quality of the foliage deposition.

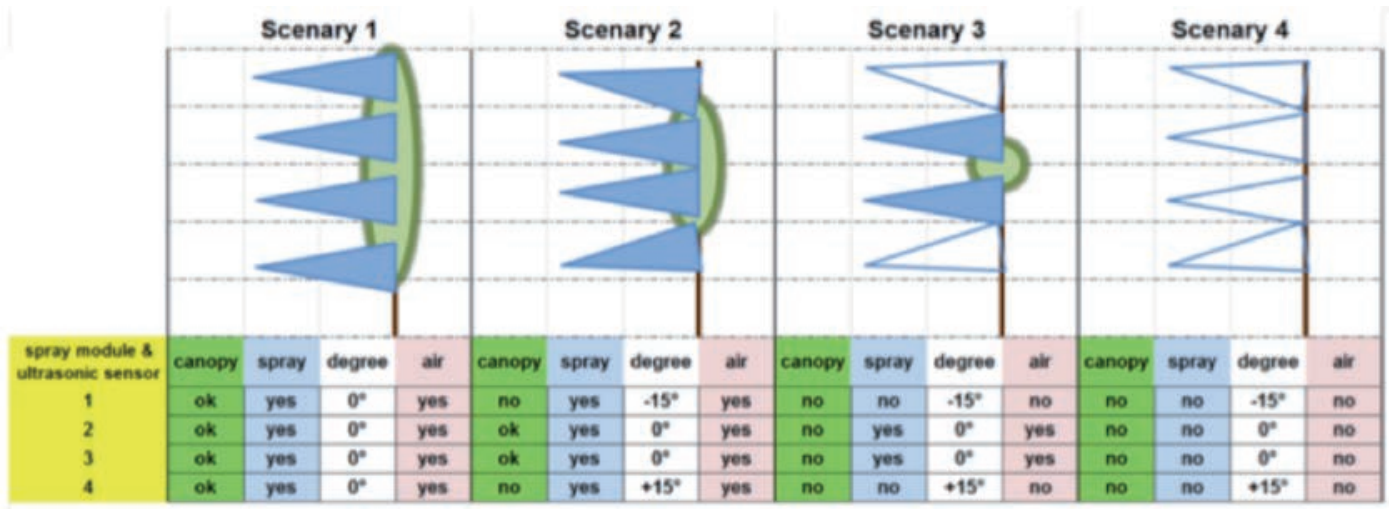

Figure 3. Rules on spray modules to adapt dosage at the canopy thickness on each band: scenario 1 corresponding to the presence of $100 \%$ of canopy $\nabla 100 \%$ dose, scenario 2 presence of $50 \% \bigotimes 70 \%$ dose, scenario 3 presence $<50 \%$ canopy thickness $\nabla 30 \%$ dose and absence of canopy 0 dose.
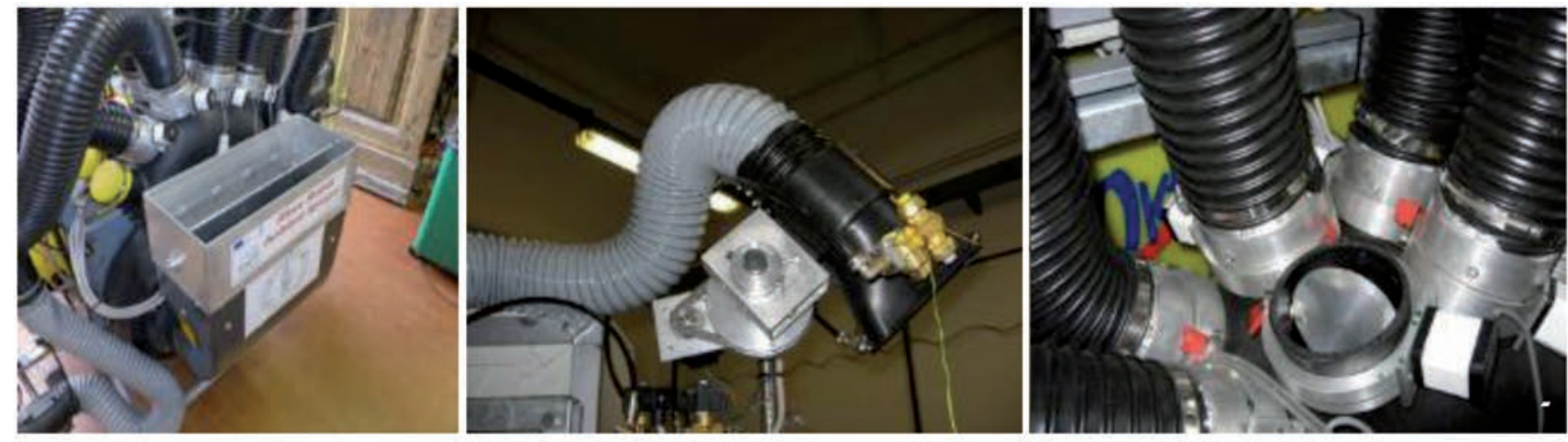

Figure 4. Devices developed for the air flow control left to right: fan inlet manifold throttle valve in the main entrance; actuation system for the upper and lower spray modules based on kinematic coupling driven by the stepper motor. This solution allows to obtain $30^{\circ}$ of rotation, thus the orientation of air flow; butterfly valves located on the exit of fan calotte to selective opening of the spray modules. 

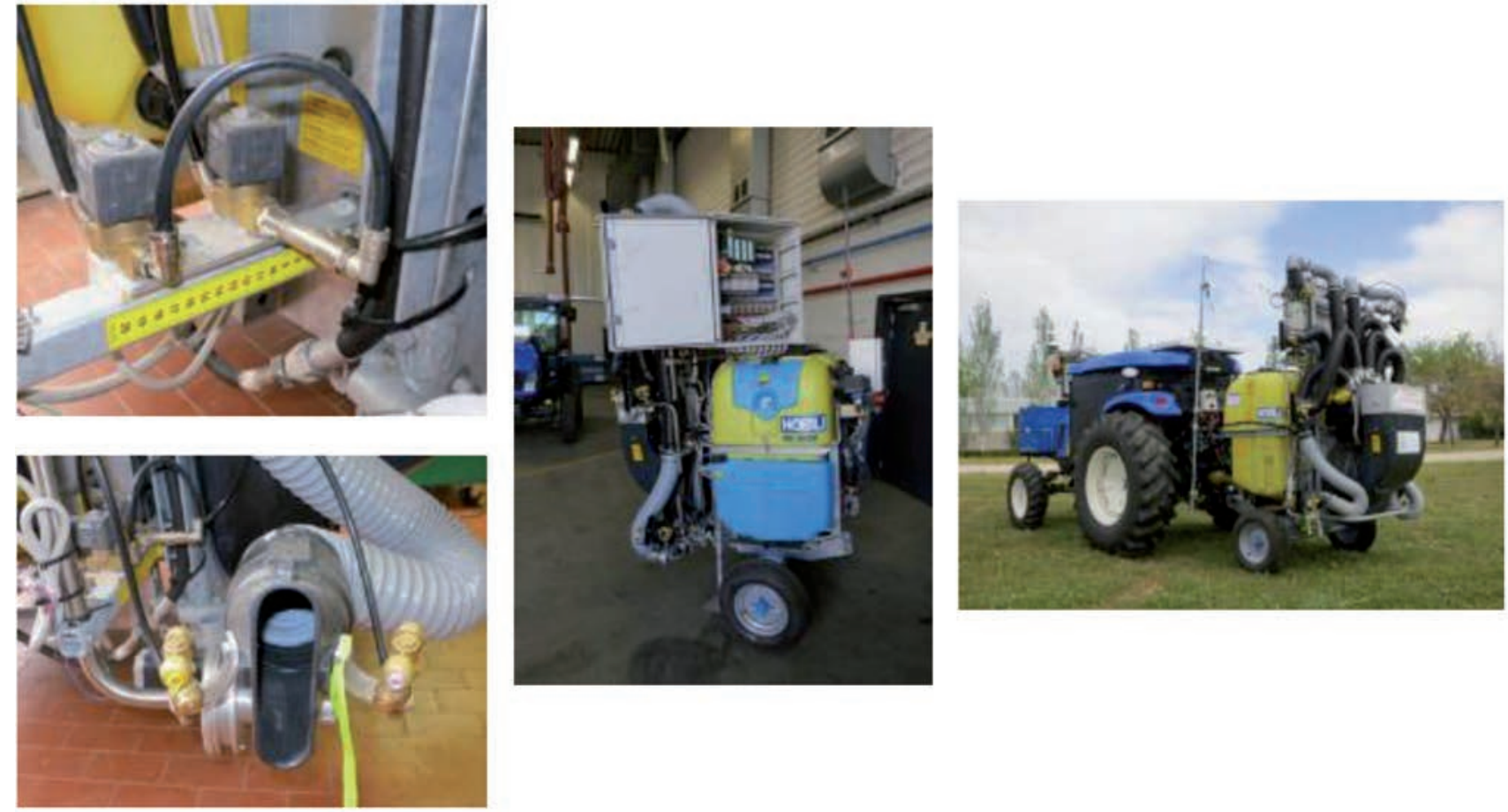

Figure 5. On the left solutions developed for the liquid flow rate control: dual nozzles on each module electronically controlled by two solenoid valves with $70 \%$ and $30 \%$ of needed flow rate on each band simultaneously open with full canopy. Centre: electronic plant, center of the low level actuation system. On the right general view of the RHEA air blast sprayer prototype coupled with the ground mobile unit.

\section{References}

Sarri D, Lisci R, Rimediotti M, Vieri M. The RHEA-project: a new generation of air-blast sprayer for tree crops pesticide application. In proceedings of the 9th European Conference on precision Agricolture, 2013 Jul 7-11, Lleida, Spain. In press in Precision Agricolture ed. Springer.

Gonzalez-de-Santos P, Vieri M, Ribeiro A, Raffaelli M, Emmi L, Fontanelli M, Rimediotti M, Frasconi C, Sarri D. \& Peruzzi A. 2011. The RHEA project: a fleet of autonomous robots for precision chemical and no chemical weed management in arable crops and oncanopy spraying in tree crops. In Proceedings of the Convegno di Medio Termine dell'Associazione Italiana di Ingegneria Agraria, Belgirate, 22-24 settembre 2011.

Vieri M, \& Spugnoli P. 1997. An high pressure injection system for pre- cision application of pesticide. BIOS Scientific Publishers Limited, Oxford 1997.

Vieri M, Lisci R, Rimediotti M, Sarri D. (2012). The innovative RHEA airblast sprayer for tree crop treatment. In proceedings of the first International Conference on Robotics and associated High-technologies and Equipment for agriculture. Application of automated systems and robotics for crop protection in sustainable precision agriculture. (RHEA 2012) Pisa, Italy, September 19-21, 2012. Pg. 93-98.

Vieri M, Sarri D, Rimediotti M, Lisci R, Peruzzi A, Raffaelli M, Fontanelli M, Frasconi C, Martelloni L. (2012). RHEA project achievement: an innovative spray concept for pesticide application to tree crops equipping a fleet of autonomous robots. Proceedings of the International Conference of Agricultural Engineering. CIGRAgEng2012 - Valencia Conference Center, 8-12 July 2012, Valencia, Spain. Report 1356. ISBN 978-84-615-9928-4. 